UVX 2012, $01011(2013)$

DOI: $10.1051 / \mathrm{uvx} / 201301011$

(C) Owned by the authors, published by EDP Sciences, 2013

\title{
Contrôle optique de la croissance et de la déformation de nanoparticules métalliques au sein de matrices mésoporeuses de $\mathrm{TiO}_{2}$
}

\author{
N. Crespo-Monteiro ${ }^{1}$, L. Nadar ${ }^{1}$, N. Destouches ${ }^{1, a}$, F. Vocanson ${ }^{1}$, L. Bois ${ }^{2}$ \\ et T. Epicier ${ }^{3}$ \\ 1 Université de Lyon, 42023 Saint-Etienne, France, CNRS, UMR 5516, Laboratoire Hubert \\ Curien, 18 rue Pr. Lauras, 42000 Saint-Etienne, France, Université de Saint-Etienne, \\ Jean-Monnet, 42000 Saint-Etienne, France \\ 2 Université de Lyon, Laboratoire Multimatériaux et Interfaces, Université Claude Bernard \\ Lyon 1, Bat Berthollet, 69622 Villeurbanne, France \\ ${ }^{3}$ Matériaux, Ingénierie et Sciences (MATEIS), UMR 5510 CNRS, Université de Lyon, \\ INSA-Lyon, 7 avenue Jean Capelle, 69621 Villeurbanne, France
}

\begin{abstract}
Résumé. Dans cet article nous caractérisons les propriétés optiques et structurales, induites sous exposition laser visible, de films mésoporeux de $\mathrm{TiO}_{2}$ amorphe contenant des nanoparticules d'argent réduites in situ de manière photo-catalytique sous rayonnement UV. Nous identifions notamment trois gammes d'intensités dans lesquelles la lumière visible induit respectivement un changement de teinte du matériau dû à une modification de la distribution de tailles des nanoparticules, un blanchiment du matériau dû à une oxydation des nanoparticules ou une cristallisation de la matrice due à l'échauffement induit par l'excitation du plasmon des nanoparticules.
\end{abstract}

\section{INTRODUCTION}

Le dioxyde de titane est un matériau bon marché, chimiquement stable et non toxique qui a été utilisé avec succès pour diverses applications comme la conversion de l'énergie solaire [1], le stockage de l'énergie électrique [2], la détection de composés chimiques [3], la dépollution de l'eau ou de l'air, ou la réalisation de surfaces auto-nettoyantes [4]. Associé à des nanoparticules (NP) métalliques il présente aussi un caractère photochromique [5]. Les propriétés photo-catalytiques de ce semiconducteur permettent dans ce cas de contrôler, sous excitation photonique, le spectre d'absorption des nanoparticules métalliques et de modifier la couleur du matériau. Ces changements de couleur réversibles résultent de variations dans la distribution de tailles des nanoparticules dues à des mécanismes d'oxydation sélective. Les NP de métal noble sont en effet connues pour être le siège d'une résonance plasmon de surface localisée induisant une absorption dans le domaine du visible dont le spectre dépend de la nature, de la forme et de la taille des nanoparticules, de l'indice de réfraction du milieu environnant et de la distance interparticule [6].

Ce type de photochromisme multicolore a été observé dans quelques matrices semiconductrices de type $\mathrm{n}$ absorbant dans la gamme de l'ultraviolet, mais la forte activité optique du $\mathrm{TiO}_{2}$ fait de lui le meilleur candidat. L'argent s'avère quant à lui être le métal noble le plus favorable à l'obtention de couleurs variées. Différents travaux rapportent un comportement photochromique avec des particules

\footnotetext{
a e-mail : nathalie.destouches@univ-st-etienne.fr
}

This is an Open Access article distributed under the terms of the Creative Commons Attribution License 2.0, which permits unrestricted use, distribution, and reproduction in any medium, provided the original work is properly cited. 


\section{Web of Conferences}

d'argent réduites au sein de matrices poreuses de $\mathrm{TiO}_{2}$ de manière photocatalytique [5,7-10], chimique $[11,12]$ ou thermique $[12,13]$ tout autant qu'avec des particules d'argent déposées par pulvérisation magnétron $[14,15]$ ou par voie électrochimique [10]. On trouve des matrices poreuses préparées à partir de NPs de $\mathrm{TiO}_{2}$ commerciales cristallisées dans la phase anatase $[5,7,8,10]$ ou bien élaborées par voie sol-gel sous forme de films mésoporeux de $\mathrm{TiO}_{2}$ amorphe [9,11-13]. Plusieurs expériences ont aussi été menées à la surface de substrats de $\mathrm{TiO}_{2}$ monocristallin [16-18]. Le contact de l'argent avec l'oxygène ou l'humidité ambiante est déterminant pour garantir la ré-oxydation des NPs, cependant une exposition trop directe rend les NP et les états colorés associés très instables à la lumière ambiante. Le contrôle de la porosité et de son homogénéité par le biais de matrices mésoporeuses semble à ce jour être une voie permettant à la fois une stabilité des états colorés photo-générés de plusieurs années et une réversibilité totale d'un état à l'autre sous flux laser [9,19].

Dans cet article nous caractérisons les propriétés optiques et structurales, induites sous exposition laser visible, de films mésoporeux de $\mathrm{TiO}_{2}$ amorphe contenant des NPs d'argent réduites in situ de manière photo-catalytique sous rayonnement UV. Nous identifions notamment trois gammes d'intensités dans lesquelles la lumière visible induit respectivement un changement de teinte du matériau dû à une modification de la distribution de tailles des NP, un blanchiment du matériau dû à une oxydation des NP ou une cristallisation de la matrice due à l'échauffement induit par l'excitation du plasmon des NP.

\section{LES FILMS AG-TIO}

Des films mésoporeux de dioxyde de titane sont élaborés par voie sol-gel selon un processus d'autoassemblage induit par évaporation (EISA) durant lequel un précurseur inorganique, l'orthotitanate de tétrabutyle (TBT) ou le tétra-isopropoxide de titane (TTIP), se condense autour d'un agent structurant. Ce dernier, le copolymère tribloc P123 dans le cas présent, est ensuite éliminé pour laisser place à une porosité de taille nanométrique $(\sim 10 \mathrm{~nm})$ homogène organisée au sein du film de $\mathrm{TiO}_{2}$. Le détail de protocoles de synthèse pouvant être utilisés se trouve par exemple dans les références $[9,11,12]$. Ces films mésoporeux, de 150 à $300 \mathrm{~nm}$ d'épaisseur, sont ensuite imprégnés d'un précurseur ionique métallique (nitrate d'argent ammoniacal), rincés puis séchés. Ils ont un aspect transparent, non diffusant et incolore. L'argent ionique présent dans le film peut être réduit sous excitation laser grâce aux propriétés photocatalytiques du $\mathrm{TiO}_{2}$, pour former des nanoparticules d'argent qui absorbent dans le visible et colorent le film. Ces dernières peuvent ensuite être excitées optiquement par l'intermédiaire de leur résonance plasmon de surface et donner lieu à un changement de couleur réversible ou à une cristallisation de la matrice environnante, selon l'intensité du flux lumineux mis en œuvre.

\section{PROPRIÉTÉS PHOTOCHROMIQUES}

\subsection{Les mécanismes}

Le photochromisme mis en œuvre dans les films $\mathrm{Ag}-\mathrm{TiO}_{2}$ repose sur la possibilité de modifier de manière réversible sous excitation photonique la distribution de tailles des nanoparticules d'argent et donc les propriétés de leur spectre de résonance plasmon de surface localisée.

Sous illumination UV, lorsque l'énergie photonique incidente est plus grande que la bande interdite du $\mathrm{TiO}_{2}$ (typ. 3,2 eV), ce denier absorbe le rayonnement incident et libère des paires électron-trou (Fig. 1). Les électrons réduisent les ions $\mathrm{Ag}(\mathrm{I})$ présents dans la porosité du film tandis que les trous oxydent les composés organiques adsorbés, comme l'eau. Les atomes d'argent réduits coalescent pour former des particules. La réduction des ions $\mathrm{Ag}(\mathrm{I})$ étant thermodynamiquement plus favorable sur des particules déjà formées que de manière isolée, et la mobilité de l'argent ionique étant grande au sein de ses matrices poreuses, l'usage de faibles intensités lumineuses (typiquement de l'ordre de quelques $\mathrm{mW} . \mathrm{cm}^{-2}$ à quelques centaines de $\mathrm{mW} . \mathrm{cm}^{-2}$ ) tend à favoriser la croissance des NP au détriment de leur nombre et donne lieu à des distributions de tailles assez larges allant de quelques nanomètres à quelques 


\section{UVX 2012}
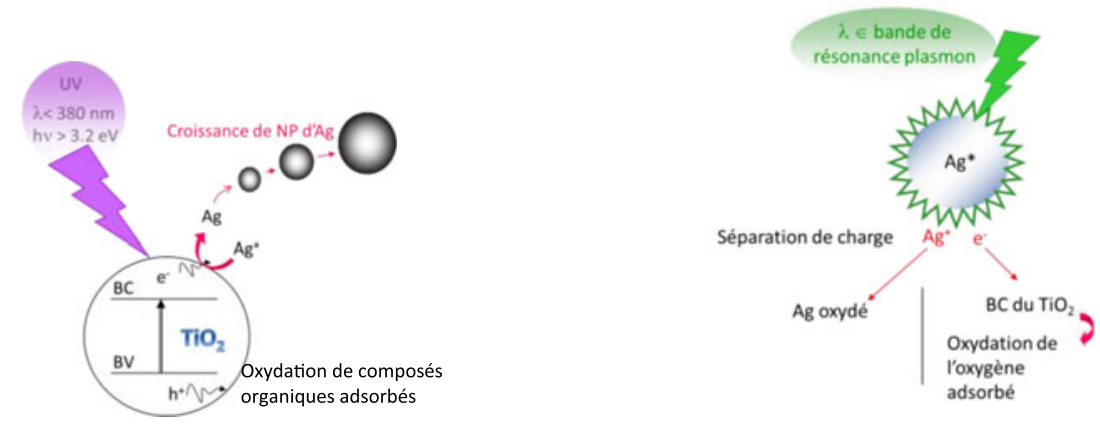

Figure 1. Schémas des mécanismes de croissance (gauche) et d'oxydation (droite) des nanoparticules d'argent sous excitation photonique.

dizaines de nanomètres. À l'inverse pour des intensités de quelques W. $\mathrm{cm}^{-2}$ à quelques $\mathrm{kW} . \mathrm{cm}^{-2}$ les NPs présentent des tailles inférieures à la taille des mésopores de la matrice et donc des distributions de tailles plus étroites. Selon la distribution de tailles obtenue, le film présente une bande d'absorption plus ou moins large dans le visible et prend une coloration orangée, brunâtre ou grisâtre $[9,12,13]$.

L'exposition du film coloré à une longueur d'onde visible permet d'exciter des électrons à la surface des nanoparticules par l'intermédiaire de l'excitation du plasmon (Fig. 1). Du fait d'une jonction Schottky à l'interface entre l'argent métallique et le $\mathrm{TiO}_{2}$, les électrons photo-excités migrent vers la bande de conduction $\mathrm{du} \mathrm{TiO}_{2}$ puis se stabilisent sur les molécules d'oxygène [10]. Les nanoparticules métalliques en contact avec la matrice d'oxyde de titane peuvent donc s'oxyder en présence d'oxygène sous illumination visible.

\subsection{Photochromisme multicolore}

Lorsque l'intensité de la lumière visible incidente est suffisamment faible, typiquement inférieure au W. $\mathrm{cm}^{-2}$, seules les nanoparticules absorbant préférentiellement à la longueur d'onde incidente s'oxydent. Une gamme de tailles disparaît donc de la distribution de tailles initiale, ce qui a pour conséquence de créer une dépression à la longueur d'onde incidente dans le spectre de l'échantillon. Cette observation mise en évidence par l'équipe du Professeur Tatsuma en 2003 sur des films élaborés à base de nanoparticules commerciales de $\mathrm{TiO}_{2}$ anatase [5], est également caractéristique des films mésoporeux dans lesquels une large distribution de nanoparticules a été obtenue sous illumination UV $(\lambda=244 \mathrm{~nm})$ de faible intensité $\left(0,282 \mathrm{~W} \cdot \mathrm{cm}^{-2}\right)$. La Figure $2 \mathrm{~b}$ montre ainsi des variations spectrales (spectrophotomètre PerkinElmer Lambda 900), observées après exposition à différentes raies d'un laser $\mathrm{Ar}-\mathrm{Kr}$ sous une intensité de $0,226 \mathrm{~W} \cdot \mathrm{cm}^{-2}$, qui témoignent d'une diminution de l'absorbance de l'échantillon au voisinage de la longueur d'onde incidente. Du fait de cette oxydation sélective en taille des nanoparticules (Fig. 2a), le nombre de petites nanoparticules tend parfois à augmenter et à créer une augmentation d'absorbance aux plus courtes longueurs d'onde (courbes verte et jaune de la Fig. 2b). Ces petites nanoparticules proviennent à la fois de la réduction de taille des nanoparticules oxydées (Fig. 2a) et de la formation de nouvelles petites particules dans le voisinage des précédentes résultant de la re-réduction spontanée des ions argent libérés par les particules oxydées [13]. Ces variations spectrales tendent à donner au film une couleur qui tend vers celle de l'illuminant (photos en insert dans la Figure 2b).

Ces couleurs sont généralement peu stables dans le temps, y compris dans l'obscurité, sauf à mettre en œuvre un traitement chimique post-insolation limitant le transfert des électrons photo-excités des nanoparticules d'argent vers l'oxygène [7]. Mais, même dans ce cas la stabilité des couleurs n'excède pas quelques jours. De ce point de vue, les films mésoporeux étudiés ici sont particulièrement 


\section{Web of Conferences}
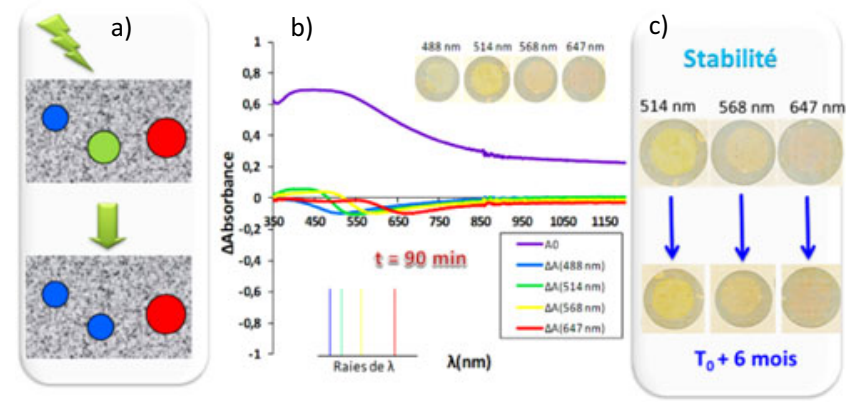

Figure 2. (a) Schéma de principe de l'oxydation sélective des nanoparticules d'argent dans une matrice de $\mathrm{TiO}_{2}$ poreuse sous excitation visible monochromatique. Les disques colorés représentent des NPs d'argent de tailles différentes absorbant préférentiellement à différentes longueurs d'onde (b) Spectre d'absorbance initial (A0) d'un film $\mathrm{Ag}-\mathrm{TiO}_{2}$ dans lequel les nanoparticules d'argent ont été formées sous exposition UV $(\lambda=244 \mathrm{~nm}$; $\left.0,282 \mathrm{~W} . \mathrm{cm}^{-2}\right)$ et variations d'absorbance $(\Delta \mathrm{A})$ mesurées après exposition à différentes longueurs d'onde visibles pendant 90 minutes avec une intensité de $0,226 \mathrm{~W} \cdot \mathrm{cm}^{-2}$. En insert, photographie de l'échantillon dans les différentes zones insolées (c) Photographies des zones colorées après les insolations laser visibles et six mois plus tard.

intéressants puisque, contrairement aux résultats habituellement rapportés dans la littérature, leur coloration est pérenne durant plusieurs mois, et ce sans avoir à subir de traitement particulier (Fig. 2c). Cette stabilité est liée au contrôle de la porosité de la matrice de $\mathrm{TiO}_{2}$ (aux alentours de $10 \mathrm{~nm}$ ) qui rend l'oxydation des nanoparticules plus difficile et nécessite l'usage d'intensités lumineuses plus élevées que celles généralement utilisées dans la littérature (quelques $\mathrm{mW} \cdot \mathrm{cm}^{-2}$ à quelques dizaines de $\mathrm{mW} . \mathrm{cm}^{-2}$ ) et voisines de l'intensité de la lumière ambiante.

\subsection{Blanchiment et cycles photochromiques}

Lorsque l'intensité dépasse quelques W.cm ${ }^{-2}$, les films $\mathrm{Ag}-\mathrm{TiO}_{2}$ tendent à blanchir sous rayonnement monochromatique. Cette propriété que nous avons d'abord mise en évidence sur des films contenant des distributions de tailles de nanoparticules étroites [9] s'avère également satisfaite pour de plus larges distributions de nanoparticules d'argent obtenues sous faible intensité UV (Fig. 3a-b). Dans ces conditions d'insolation, toutes les tailles de nanoparticules sont donc oxydées simultanément, qu'elles absorbent fortement ou moins fortement le rayonnement incident. Ce blanchiment est réversible, le film retrouvant une coloration brun-gris caractéristique d'une large distribution de nanoparticules d'argent sous insolation UV. La Figure $3 c$ montre en outre que ce cycle de coloration/blanchiment est répétable plusieurs fois sans perte importante de contraste. On notera que les NPs d'argent se forment essentiellement dans les 100 premiers nanomètres du film mesoporeux (Fig. 3b). La partie imaginaire de la fonction diélectrique du $\mathrm{TiO}_{2}$ est en effet très importante à $244 \mathrm{~nm}$ et conduit à une absorption de $90 \%$ du flux lumineux incident en quelques dizaines de nanomètres seulement.

\section{CRISTALLISATION INDUITE PAR LASER VISIBLE}

Pour des intensités visibles dépassant la centaine de W. $\mathrm{cm}^{-2}$, l'absorption des nanoparticules métalliques induit un échauffement local suffisant pour cristalliser la matrice de $\mathrm{TiO}_{2}$ environnante au sein de domaines allant de quelques nanomètres à quelques centaines de nanomètres de long (Fig. 4a-b). Cette cristallisation peut être induite sous rayonnement monochromatique ; le seuil en intensité requis pour l'engendrer dépend de la longueur d'onde incidente et du spectre d'absorbance initial. Elle induit un effondrement de la mésostructure et une forte diminution de l'épaisseur du film qui peut être utilisée pour graver des motifs sur le film en contrôlant la profondeur de gravure à une dizaine de nanomètres 

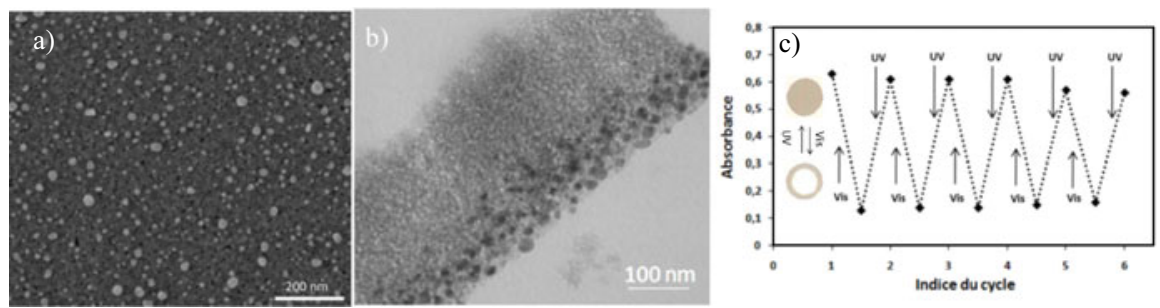

Figure 3. Images d'un film $\mathrm{Ag}-\mathrm{TiO}_{2}$ présentant une large distribution de nanoparticules métalliques obtenues par microscopie électronique à balayage (FEI Nova nanoSEM 200) en vue de dessus (a) ou en transmission (Hitachi H-800) sur une section droite du film (b). (c) Variations de l'absorbance à la résonance mesurées au cours de cycles photochromiques sur ce film soumis successivement à un laser émettant à $244 \mathrm{~nm}\left(\mathrm{I}=0,919 \mathrm{~W} \cdot \mathrm{cm}^{-2}\right)$ pendant $5 \mathrm{~min}$ puis à un laser émettant à $488 \mathrm{~nm}\left(\mathrm{I}=7,07 \mathrm{~W} \cdot \mathrm{cm}^{-2}\right)$ pendant $30 \mathrm{~min}$. En insert à gauche : photos du film après formation des nanoparticules sous UV (haut) et après blanchiment à $488 \mathrm{~nm}$ (bas).
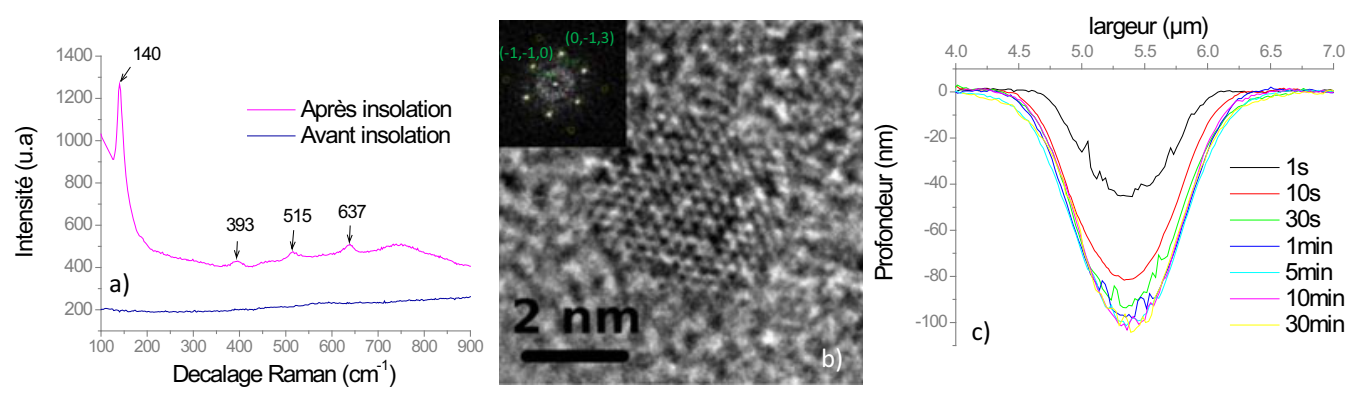

Figure 4. Nanocristallisation d'un film $\mathrm{Ag}-\mathrm{TiO}_{2}$ sous insolation à $488 \mathrm{~nm}$ avec une intensité de $90 \mathrm{~kW} \cdot \mathrm{cm}^{-2}$ (a) Spectres Raman mesurés avant et après insolation montrant la croissance d'une phase anatase. (b) Image de microscopie électronique en transmission haute résolution (METHR_JEOL 2010F) de nanoparticules de $\mathrm{TiO}_{2}$ cristallisées dans la phase anatase. (c) Profils de trous formés par une cristallisation locale sous faisceau focalisé $\left(\lambda=488 \mathrm{~nm}\right.$ et $\left.\mathrm{I}=10 \mathrm{~kW} \cdot \mathrm{cm}^{-2}\right)$ mesurés par microscopie à force atomique.

près (Fig. 4c). Ces motifs sont pérennes et résistent à des montées en température supérieures à $900^{\circ} \mathrm{C}$. En revanche, le film ainsi nanocristallisé ne présente plus de propriétés photochromiques. Il peut être noté qu'en l'absence d'argent dans les films, tout rayonnement visible incident ayant une intensité inférieure à $3 \mathrm{MW} \cdot \mathrm{cm}^{-2}$ (limite expérimentale) n'a aucun effet sur le film de $\mathrm{TiO}_{2}$ mésoporeux.

\section{CONCLUSION}

Les films mésoporeux d'oxyde de titane imprégnés de sel d'argent présentent un caractère photochromique réversible dont les états colorés sont stables sur de longues périodes en l'absence d'excitation photonique dont l'intensité dépasse quelques dizaines de $\mathrm{mW} \cdot \mathrm{cm}^{-2}$. Ces états colorés sont dus à la formation de nanoparticules d'argent dans les films dont la distribution de tailles peut être ajustée en fonction des conditions d'insolation. Les films peuvent prendre différentes couleurs sous exposition visible monochromatique en fonction de la longueur d'onde mise en jeu. Ces couleurs qui résultent d'une oxydation sélective de certaines tailles de nanoparticules d'argent ne sont obtenues que pour des intensités inférieures au W.cm ${ }^{-2}$. Pour des intensités comprises typiquement entre le W.cm ${ }^{-2}$ et la centaine de W. $\mathrm{cm}^{-2}$, tout rayonnement monochromatique dont la longueur d'onde est comprise dans la bande de résonance plasmon localisée des nanoparticules tend à blanchir le film, c'est-à-dire à oxyder l'ensemble des nanoparticules. Les mécanismes de coloration/blanchiment sont réversibles et répétables 


\section{Web of Conferences}

sur un même film. Lorsque l'intensité visible incidente dépasse la centaine de W.cm ${ }^{-2}$, l'échauffement photo-induit des nanoparticules métalliques devient suffisant pour nanocristalliser la matrice de $\mathrm{TiO}_{2}$ et engendre une diminution de l'épaisseur du film qui peut être contrôlée très précisément en ajustant l'intensité, la longueur d'onde du faisceau incident ou le temps d'exposition. Cette densification locale du film peut être utilisée pour réaliser du photo-marquage pérenne résistant à des montées en températures de plusieurs centaines de degrés.

\section{Remerciements}

Les auteurs remercient l'ANR pour son soutien financier dans le cadre du projet UPCOLOR n JCJC 201010021 et le CLYM (clym.fr) pour l'accès au METHR JEOL 2010F.

\section{Références}

[1] M. Gratzel, Nature 414, 338 (2001)

[2] J. Wang, Y. Zhou, Y. Hu, R. O’Hayre, Z. Shao, J. Phys. Chem. C 115, 2529 (2011)

[3] Wang, C.X., Yin, L.W., Zhang, L.Y., Gao, R. J. Phys. Chem. C 114, 4408 (2010)

[4] K. Hashimoto, H. Irie, A. Fujishima, AAPPS Bulletin December 17, 12 (2007)

[5] Y. Ohko, T. Tatsuma, T. Fujii, K. Naoi, C. Niwa, Y. Kubota, A. Fujishima, Nat. Mater. 2, 29 (2003)

[6] R. Jin, Y. Cao, C.A. Mirkin, K.L. Kelly, G.C. Schatz, J.G. Zheng, Science 294, 1901 (2001)

[7] K. Naoi, Y. Ohko, T. Tatsuma, Chem. Comun. 10, 1288 (2005)

[8] J. Preclíková, F. Trojánek, P. Nemec, P. Malý, J. Phys. Status Solidi (C) 5, 3496 (2008)

[9] N. Crespo-Monteiro, N. Destouches, L. Bois, F. Chassagneux, S. Reynaud, T. Fournel, Adv. Mater. 22, 3166 (2010)

[10] K. Kawahara, K. Suzuki, Y. Ohko, T. Tatsuma, Phys. Chem. Chem. Phys. 7, 3851 (2005)

[11] L. Bois, F. Chassagneux, Y. Battie, F. Bessueille, L. Mollet, S. Parola, N. Destouches, N. Toulhoat, N. Moncoffre, Langmuir 26, 1199 (2010)

[12] L. Nadar, R. Sayah, F. Vocanson, N. Crespo-Monteiro, A. Boukenter, S. Sao Joao, N. Destouches, Photochem. Photobiol. Sci. 10, 1810 (2011)

[13] N. Crespo-Monteiro, N. Destouches, L. Nadar, S. Reynaud, F. Vocanson, J.-Y. Michalon, Appl. Phys. Lett. 99, 173106 (2011)

[14] C. Dahmen, A.N. Sprafke, H. Dieker, M. Wuttig, G. von Plessen, Appl. Phys. Lett. 88, 011923 (2006)

[15] J. Okumu, C. Dahmen, A.N. Sprafke, M. Luysberg, M., G. von Plessen, M. Wuttig, J. Appl. Phys. 97, 094305 (2005)

[16] K. Matsubara, T. Tatsuma Adv. Mater. 19, 2802 (2007)

[17] K. Matsubara, K.L. Kelly, N. Sakai, T. Tatsuma, Phys. Chem. Chem. Phys. 10, 2263 (2008)

[18] K. Matsubara K.L. Kelly, N. Sakai, T. Tatsuma, J. Mater. Chem. 19, 5526 (2009)

[19] N. Crespo-Monteiro, N. Destouches, T. Fournel, Appl. Phys. Express 5, 075803 (2012) 